\title{
Bilateral Diffuse Pseudoangiomatous Stromal Hyperplasia (PASH) Causing Gigantomastia in a 33-Year- Old Pregnant Woman: Case Report
}

\author{
Natalia Krawczyk $^{a}$ Tanja Fehm ${ }^{a}$ Eugen Ruckhäberle ${ }^{a}$ Svjetlana Mohrmann $^{a}$ \\ Jasmin Riemer $^{\mathrm{b}}$ Stefan Braunstein ${ }^{\mathrm{b}}$ Jürgen Hoffmann ${ }^{\mathrm{a}}$ \\ a Department of Obstetrics and Gynaecology, Heinrich-Heine University, Düsseldorf, Germany; \\ ${ }^{b}$ Department of Pathology, Heinrich-Heine University, Düsseldorf, Germany
}

\section{Established Facts}

- PASH is a benign proliferation of mammary stroma.

- The main clinical differential diagnosis is fibroadenoma or phylloid tumour and histologically a low grade angiosarcoma.

Novel Insights

- Diffuse PASH can occur during pregnancy, causing massive breast enlargement and requiring bilateral mastectomy.

\section{Keywords}

Diffuse pseudoangiomatous stromal hyperplasia .

PASH · Pregnancy · Breast - Mastectomy

\section{Summary}

Pseudoangiomatous stromal hyperplasia (PASH) is a benign proliferation of mammary stroma mostly described as an incidental microscopic finding. Clinically, it can manifest as a palpable, well-circumscribed breast mass or in rare cases as a diffuse bilateral process causing massive breast enlargement. The most postulated theory for aetiology of this condition is hormonal stimulation of mammary myofibroblasts, particularly by progesterone. A definite diagnosis of PASH is based on typical pathological findings like stromal hyperplasia and empty slit-like channels positive for myofibroblastic and negative for endothelial markers. The main clinical differential diagnosis is a fibroadedoma or phylloid tumour, and his- tologically a low-grade angiosarcoma. There are less than 200 cases of tumorous PASH and less than 20 of diffuse PASH reported so far. Here we present a case report of huge diffuse PASH, that is, to our knowledge, the first in a pregnant woman.

(ㄷ) 2016 S. Karger GmbH, Freiburg

\section{Introduction}

Pseudoangiomatous stromal hyperplasia (PASH) is a benign proliferation of the breast stroma mostly described as an incidental pathologic finding associated with several breast entities like fibrocystic changes, fibroadenomas, gynaecomastia or breast cancer [1]. This condition rarely occurs as a clinically apparent mammary lesion originally described in 1986 by Vuitch et al. [2]. A typical clin-

\section{KARGER}

() 2016 S. Karger GmbH, Freiburg
Dr. med. Natalia Krawczyk

Department of Obstetrics and Gynaecology Heinrich-Heine University

Moorenstrasse 5, 40225 Düsseldorf, Germany natalia_krawczyk@yahoo.de 

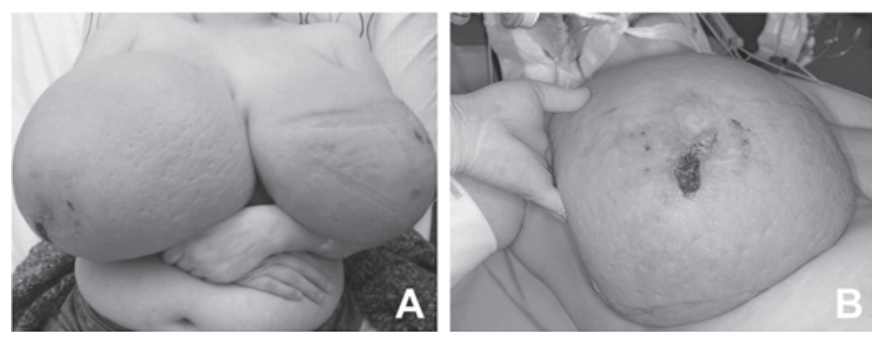

Fig. 1. A Asymmetric breast enlargement within first trimester of pregnancy. B Painful skin ulcerations.

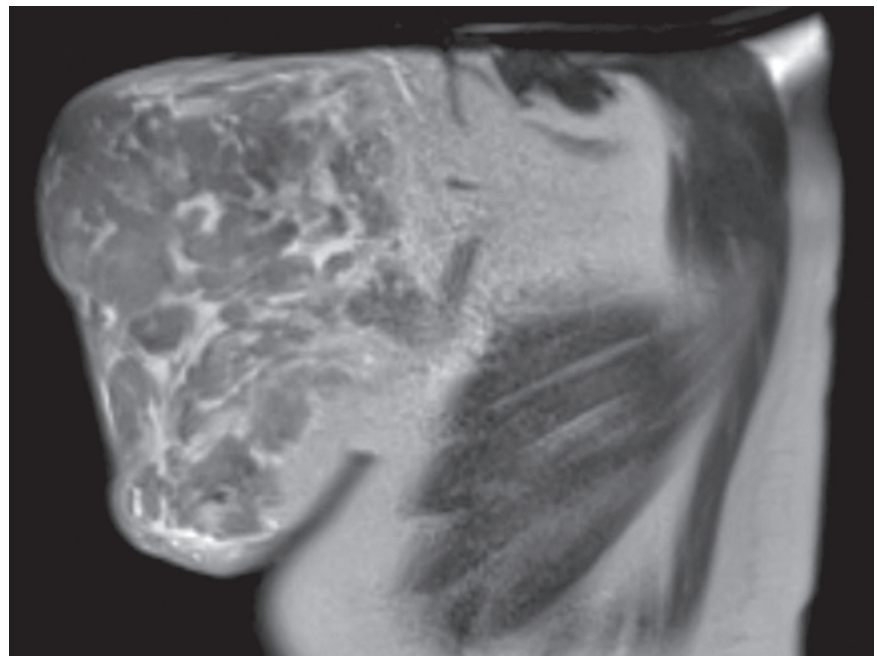

Fig. 2. Sagittal magnetic resonance imaging of the left breast, showing massive lymphoedema, distinctive thickening of the skin and subcutaneous tissue. There are no circumscribed mass lesions.

ical manifestation is a unilateral breast mass, mostly mimicking a fibroadenoma (nodular PASH). In very rare cases PASH presents as bilateral diffuse process leading to rapid breast enlargement without discrete nodular mass (diffuse PASH). Less than 200 cases of nodular PASH and less than 20 of rapidly growing diffuse PASH have been reported in the literature so far, to our knowledge, none of them in a pregnant woman [3-5].

\section{Case Report}

A 33-year-old primigravida at 14 weeks of dichorionic diamniotic twin pregnancy presented at our department with massive bilateral breast enlarge ment within the first trimester of gestation. The patient reported breast tenderness, back pain with movement limitation and respiratory impairment. Her previous medical conditions included bronchial asthma. Physical examination revealed bilateral asymmetric gigantomastia, with the right breast being larger than the left, diffuse lymphoedema and multiple skin ulcerations (fig. 1). No isolated mammary nodules were palpable. Bilateral diagnostic excisional biopsies performed at another hospital showed diffuse PASH of both breasts. The patient was referred to our certified breast cancer centre for further evaluation. A breast ultrasound showed increased breast density with pronounced lymphoedema without circumscribed nodular lesions. No local lymphadenopathy was found. A breast magnetic resonance imaging demonstrated massive lymphoedema with distinctive thickening of the skin and subcutaneous tissue and no circumscribed mass lesions (fig. 2). Due to rapid pro-
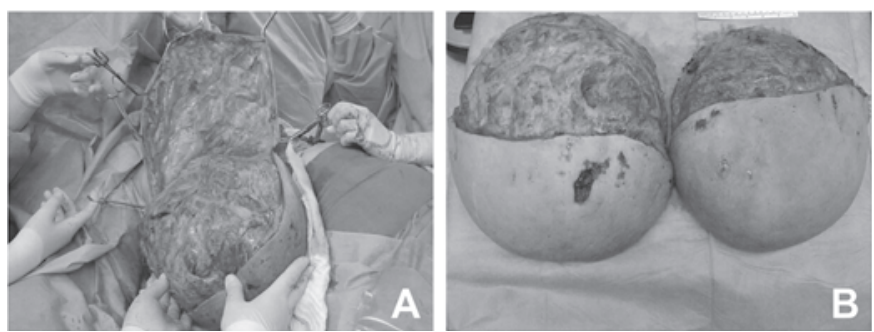

Fig. 3. A Skin-sparing mastectomy at 16 weeks of pregnancy. B Bilateral mastectomy specimens.

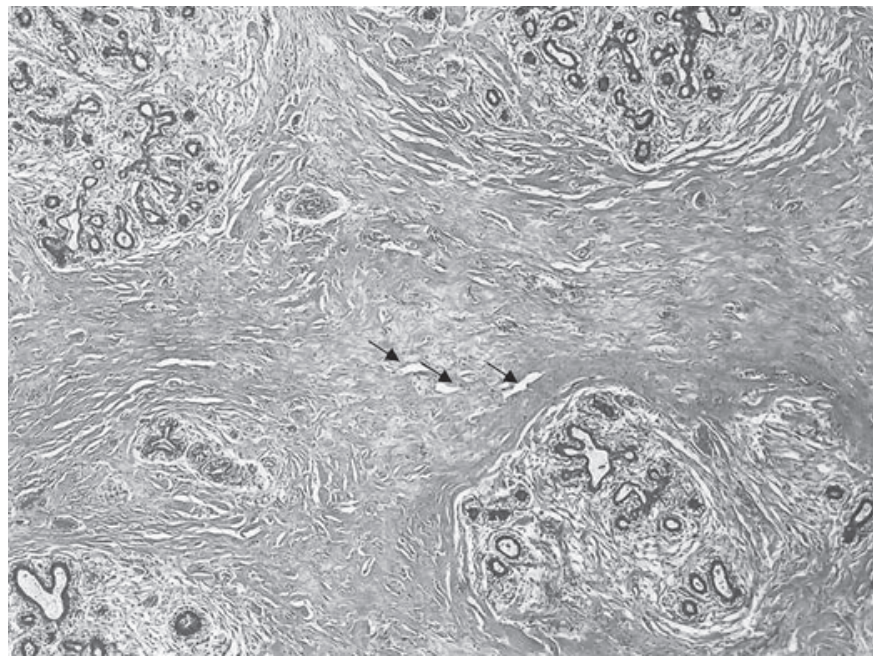

Fig. 4. Stromal hyperplasia and slit-like spaces (marked with arrows) lined by endothelial-like spindle cells.

gression of the macromastia, resulting in worsening of the respiratory impairment and pain, a bilateral skin-sparing mastectomy with immediate reconstruction was performed at 16 weeks of the pregnancy. Bilateral mastectomy specimens (7,273 g right and 5,504 g left) were removed (fig. 3). Histological tissue examination revealed typical characteristics of PASH-like stromal hyperplasia and slit-like spaces lined by endothelial-like spindle cells (fig. 4). Moreover, stromal oedema, necrosis, fibrous mastopathy, adedosis and epidermoid cysts were found in both breasts. The further course of pregnancy was uneventful; healthy twins were delivered by caesarean section at 38 weeks of gestation.

\section{Discussion}

PASH represents hyperplasia of mammary stroma commonly accompanying benign and malignant breast entities. As demonstrated by Ibrahim et al. [1], incidental microscopic PASH can be found in up to $23 \%$ of consecutive breast specimens. In contrast, clinically manifest PASH as a main pathological finding is a rare entity. Typically it forms a solitary, unilateral, well-circumscribed mass that mostly mimics a fibroadenoma or phylloid tumour. In rare cases it can appear bilaterally forming diffuse multiple tumours and leading to massive asymmetric breast enlargement. On rare occasions this phenomenon can occur without formation of the obvious tumour mass, as presented in our patient [6]. 
Although no definite mechanism of PASH has yet been identified, the most accepted theory for the aetiology and pathogenesis of this condition is a hormonal stimulation of mammary myofibroblasts. This hypothesis is supported by the fact that the majority of PASH cases show oestrogen receptor and most of all progesterone receptor positivity of stromal cells $[7,8]$. This findings correspond to clinical observations that PASH mostly occurs in premenopausal women or postmenopausal women who received hormone replacement therapy. A dominant role of progesterone could also explain the rapid breast enlargement in our patient during first trimester of the pregnancy. However, there are also rare reports of PASH in children or postmenopausal women with no exogenous hormonal influence [9-11].

While the clinical manifestation of PASH may vary widely between patients, definite diagnosis is based on typical histological findings, such as empty slit-like spaces formed in acellular dense collagenous stroma lined by spindle cells and imitating blood vessels [6]. Immunohistochemical positivity for CD34, vimentin or smooth muscle actin supports the myofibroblastic origin of these cells, whereas negativity for endothelial markers such as CD31 and von Willebrand factor helps the distinction from low-grade angiosarcoma, the main microscopic differential diagnosis of PASH $[1,6,8]$.

$\mathrm{PASH}$ is a benign condition with excellent prognosis. Incidental PASH does not require any additional treatment and tumorous PASH is treated by local excision with clear margins. However, the very rare diffuse PASH with massive breast enlargement requires uni- or bilateral mastectomy as in our patient and other reported cases [3-5]. The risk of recurrence after surgical excision is up to $22 \%[6,12]$. There is no established conservative therapy for $\mathrm{PASH}$, although a single case of successful treatment with tamoxifen has been reported by Pruthi and colleagues [13]. PASH is not considered a premalignant lesion or a risk factor for a breast cancer $[12,14]$.

\section{Disclosure Statement}

The authors declare that they have no conflicts of interest.

\section{References}

1 Ibrahim RE, Sciotto CG, Weidner N: Pseudoangiomatous hyperplasia of mammary stroma. Some observations regarding its clinicopathologic spectrum. Cancer 1989;63:1154-1160.

2 Vuitch MF, Rosen PP, Erlandson RA: Pseudoangiomatous hyperplasia of mammary stroma. Hum Pathol 1986;17:185-191.

3 Yoo K, Woo OH, Yong HS, et al.: Fast-growing pseudoangiomatous stromal hyperplasia of the breast: Report of a case. Surg Today 2007;37:967-970.

4 Ryu EM, Whang IY, Chang ED: Rapidly growing bilateral pseudoangiomatous stromal hyperplasia of the breast. Korean J Radiol 2010;11:355-358.

5 Dai H, Connor C, Cui W, et al.: Bilateral diffuse tumorous pseudoangiomatous stromal hyperplasia: A case of bilateral mastectomy in a 29-year-old woman. Case Rep Pathol 2014;2014:250608.

6 Virk RK, Khan A: Pseudoangiomatous stromal hyperplasia: An overview. Arch Pathol Lab Med 2010;134: 1070-1074.
7 Anderson C, Ricci A Jr, Pedersen CA, Cartun RW: Immunocytochemical analysis of estrogen and progesterone receptors in benign stromal lesions of the breast. Evidence for hormonal etiology in pseudoangiomatous hyperplasia of mammary stroma. Am J Surg Pathol 1991;15:145-149.

8 Powell CM, Cranor ML, Rosen PP: Pseudoangiomatous stromal hyperplasia (PASH). A mammary stromal tumor with myofibroblastic differentiation. Am J Surg Pathol 1995, 19:270-277.

9 Shehata BM, Fishman I, Collings MH, et al.: Pseudoangiomatous stromal hyperplasia of the breast in pediatric patients: An underrecognized entity. Pediatr Dev Pathol 2009; 12:450-454.

10 Singh KA, Lewis MM, Runge RL, Carlson GW: Pseudoangiomatous stromal hyperplasia. A case for bilateral mastectomy in a 12-year-old girl. Breast J 2007; 13: 603-606.
11 Jones KN, Glazebrook KN, Reynolds C: Pseudoangiomatous stromal hyperplasia: Imaging findings with pathologic and clinical correlation. AJR Am J Roentgenol 2010;195:1036-1042.

12 Ferreira M, Albarracin CT, Resetkova E: Pseudoangiomatous stromal hyperplasia tumor: A clinical, radiologic and pathologic study of 26 cases. Mod Pathol 2008;21:201-207.

13 Pruthi S, Reynolds C, Johnson RE, Gisvold JJ: Tamoxifen in the management of pseudoangiomatous stromal hyperplasia. Breast J 2001;7:434-439.

14 Degnim AC, Frost MH, Radisky DC, et al.: Pseudoangiomatous stromal hyperplasia and breast cancer risk. Ann Surg Oncol 2010;17:3269-3277. 